\title{
Ultrasound-guided psoas compartment and sciatic nerve blocks for pain management of hind limb procedures in the alpaca (Vicugna pacos)
}

\author{
Andrew Foster ${ }^{*}$ Alex McSloy and Paolo Monticelli \\ Department of Clinical Science and Services, Royal Veterinary College, University of London, Hatfield, \\ Hertfordshire, UK
}

\begin{abstract}
Background: Loco-regional anesthetic techniques are considered important in the multimodal approach to analgesia in both human and veterinary medicine. No such techniques are described in the alpaca, bar the use of epidural. This is in part due to the lack of anatomical description for this species. While this limitation exists, the use of ultrasound guidance makes peripheral nerve blocks a viable possibility in the alpaca.

Case description: A 12-month-old alpaca was referred for the treatment of a septic left tarso-crural joint. Due to a poor prognosis, amputation of the limb under general anesthesia was performed. Sciatic and psoas compartment blocks were attempted with the ultrasound-guided injection of ropivacaine prior to the start of the procedure. While the femoral nerve was possible to visualize, no obturator nerve was identified. A 5-year old alpaca was also referred for a left hind lateral claw removal, due to a squamous cell carcinoma. The sciatic nerve block was performed prior to the claw amputation under general anesthesia. No changes in heart and respiratory rate, or blood pressure suggestive of nociception, were observed in either of these cases. At the end of both the procedures, the patients experienced uneventful recoveries characterized by the ability to maintain the standing position, interest in food, and normal behavior.

Conclusion: This report identifies the ease of performance and the challenges encountered using the ultrasoundguided psoas compartment and sciatic nerve blocks in the alpaca. It is our hope that this report encourages the use of ultrasound-guided loco-regional techniques in this species.
\end{abstract}

Keywords: Alpaca, Loco-regional anesthesia, Psoas compartment block, Sciatic nerve block, Ultrasound-guided.

\section{Introduction}

With an ever-increasing number of alpacas kept worldwide as both pets and farm animals, more complicated surgical procedures are being performed and hence, an improvement in their anesthetic and analgesic management is required. While neuroaxial anesthesia is described in camelid species (Tee et al., 2005; Garcia Pereira et al., 2006; Abrahamsen, 2009), peripheral nerve blocks (PNBs) are not. The main advantages of PNBs over neuroaxial anesthesia are the production of unilateral motor blockade, decreased incidence of intraoperative hypotension, and urinary retention after surgery, while providing analgesia that is at least comparable to that of epidural administration of local anesthetics (LA) (Campoy et al., 2012). Alpacas are particularly nervous animals and this can make them challenging to manage in a clinical environment (Anderson, 2013). It is reasonable to hypothesize that the administration of unilateral nerve blocks, as opposed to neuraxial block (affecting motor function bilaterally), may assist in achieving ambulation more rapidly following orthopedic surgery, improving recovery, and subsequently patient welfare. Prompt ambulation may also decrease the risk of myopathies and neuropathies of the limbs that even if not frequently reported in camelids, are potential complications for farm animal species (HuiChu, 2014).

Loco-regional anesthesia is widely accepted to provide intra- and post-operative analgesia, with a decrease in requirement for volatile anesthetics and systemic analgesia in both humans (Touray et al., 2008) and dogs (Campoy et al., 2012). Indeed, the use of PNBs is a popular choice in small animal anesthesia for the provision of analgesia to patients undergoing orthopedic surgeries. The most commonly used technique is the administration of local anesthetics in close proximity to the sciatic (SN) and femoral nerve (FN) in order to provide motor and sensory blockade of the two main nerves of the hind limb. These are considered useful for procedures of the stifle and distal limb. For FN block, the most commonly used approach is through the medial femoral triangle, an area that is delineated by the pectineus muscle caudally, the sartorius muscle cranially, and the iliopsoas muscle (IPM) proximally (Campoy and Mahler, 2013). These techniques have been successfully reported in a number of species, including the dog, guinea pig, rabbit, goat, calf, wallaby, and raptor (Shilo et al., 2010; Adami et al., 
2011; Aguiar et al., 2014; d'Ovidio et al., 2014; 2015; Viscasillas et al., 2015; Monticelli et al., 2016).

More recently, there has been interest in the psoas compartment approach (PsC) to the $\mathrm{FN}$ in small animals (Haro et al., 2012; Portela et al., 2013; Tayari et al., 2017). This technique aims to block both the $\mathrm{FN}$ and the obturator nerve $(\mathrm{ON})$, utilizing their close trajectory from the lumbar plexus as they travel through the IPM. This approach offers a number of advantages compared to blocking the FN alone with an inguinal approach: 1) the ON provides sensory innervation to the stifle that when blocked, reduces the overall requirement for perioperative systemic analgesia in humans (McNamee et al., 2002) and dogs (Vettorato et al., 2013); 2) the femoral artery is avoided with this approach; 3 ) the patient can remain in the same lateral recumbency as that utilized to perform the SN block, an aspect that may be of particular relevance for patients with fractures.

This case report discusses performing these PNBs and the challenges encountered in alpacas undergoing orthopedic surgery of the hind limbs.

\section{Case Details}

\section{Case 1}

A 12-month-old female pet Huacaya Alpaca (Vicugna pacos) weighing $20 \mathrm{~kg}$ was referred to the Royal Veterinary College for the investigation of hind limb lameness of 9 days duration.

Based on CT images acquired and the results of cytological examination of intra-articular fluid, a diagnosis of a septic left tarso-crural joint was made. Due to a poor prognosis, amputation of the limb at the level of mid femur under general anesthesia was performed.

Pre-anesthetic examination was deemed unremarkable. The patient was starved for 12 hours and access to water withheld for 8 hours prior to surgery. A 16-gauge catheter was placed in the right jugular vein, under manual restraint only.

After pre-medication with $0.01 \mathrm{mg} / \mathrm{kg}$ buprenorphine (Buprecare ${ }^{\circledR} 0.3 \mathrm{mg} / \mathrm{ml}$, Animalcare, York, UK), 0.2 $\mathrm{mg} / \mathrm{kg}$ midazolam (Hypnovel ${ }^{\circledR} 5 \mathrm{mg} / \mathrm{ml}$, Roche, Basel, Switzerland), and $0.1 \mathrm{mg} / \mathrm{kg}$ xylazine (Chanazine ${ }^{\circledR}$ $10 \%$, Chanelle Pharma Group, Loughrea, Ireland) intravenously (IV); the patient was induced with 3 $\mathrm{mg} / \mathrm{kg}$ ketamine (Ketamidor ${ }^{\circledR} 100 \mathrm{mg} / \mathrm{ml}$, Chanelle Pharma Group, Loughrea, Ireland) IV and intubated with a $7 \mathrm{~mm}$ ID cuffed endotracheal tube via direct laryngoscopic visualization. The alpaca was connected to a circle rebreathing system (Mallard $2850 \mathrm{~TB}$, USA) and anesthesia was maintained with isoflurane in oxygen and air (0.8 and 0.2 , respectively). Hartmann's solution was infused at $2 \mathrm{ml} / \mathrm{kg}$ /hour. A $\mathrm{S} / 5$ Datex-Ohmeda multiparameter module was used to monitor physiological parameters. Heart rate (HR), respiratory rate (RR), pulse oximetry, capnography, spirometry, inspiratory and expiratory fraction of gases, temperature, and non-invasive blood pressure (BP) were recorded every 5 minutes. Mechanical ventilation was initiated immediately following the start of volatile anesthesia, with tidal volume and respiratory rate adjusted to maintain normocapnia.

For provision of the PNBs, a total of $0.3 \mathrm{ml} / \mathrm{kg}(2.25$ $\mathrm{mg} / \mathrm{kg}$ ) of ropivacaine (Naropin ${ }^{\circledR}$ 0.75\%, AstraZeneca, Cambridge, UK) was drawn up, with $0.1 \mathrm{ml} / \mathrm{kg}$ utilized for the SN block and the remaining $0.2 \mathrm{ml} / \mathrm{kg}$ utilized for the PsC block. The ultrasound guided SN block was performed using a portable ultrasound system $\left(\right.$ MyLab $^{\mathrm{TM}}$ 30, Esoate, Genova, Italy) as described by Echeverry et al. (2010), using a 22G $63 \mathrm{~mm}$ Quincke spinal needle (Figs. 1 and 2). With the transducer placed distal to the trochanter major in a transverse plane, the SN was identified as two ellipsoid structures surrounded by a thin hyperechoic rim deep to the biceps femoris muscle. The needle was advanced inplane and the nerve approached in a transverse view. The LA solution was injected after aspirating, to rule out intravascular needle placement. Correct positioning was confirmed by spread of LA solution around the circumference of the nerve.

With the patient remaining in right lateral recumbency, the psoas compartment block was performed using the technique described by Tayari et al (2017). The ultrasound transducer was placed perpendicularly to the long axis of the spine just cranial to the wing of the ilium in the lumbar area, in a torso-ventral position. After visualization of the IPM and the FN (a rounded hypoechoic structure with a thin hyperechoic rim), the transducer was rotated $45^{\circ}$ dorsally on its short axis and oriented caudally, maintaining the ultrasound view of the IPM and FN. The FN was the only nervous structure that was possible to recognize in the psoas compartment and so the ON was not identified. The needle was then advanced in-plane, until it was located in close proximity to the FN (Figs. 3 and 4). The LA solution was injected after aspirating, to rule out intravascular needle placement. Correct positioning was confirmed by spread of local anesthetic around the FN.

Limb amputation was then performed. During this time, the patient experienced mild hypotension with a mean arterial pressure of $60 \mathrm{mmHg}$. An IV bolus of $0.1 \mathrm{mg} / \mathrm{kg}$ ephedrine (Ephedrine Hydrochloride, Martindale Pharmaceuticals, Buckinghamshire, UK) and norepinephrine (Noradrenaline $4 \mathrm{mg} / \mathrm{ml}$, Hospira, Hurley, UK) continuous rate infusion of $0.1 \mathrm{mcg} / \mathrm{kg} /$ min were required to maintain the mean blood pressure (BP) above $70 \mathrm{mmHg}$. End-tidal isoflurane (ETiso) concentration ranged from $1.1 \%$ to $1.3 \%$ throughout. General anesthesia lasted 3 hours and 25 minutes. At the end of anesthesia, the patient was transferred to the recovery box.

Recovery was considered smooth with full ambulation regained within 90 minutes following the end of gaseous anesthesia, despite the low body temperature $\left(32.7^{\circ} \mathrm{C}\right)$. Post-operative analgesia was further provided with 0.01 


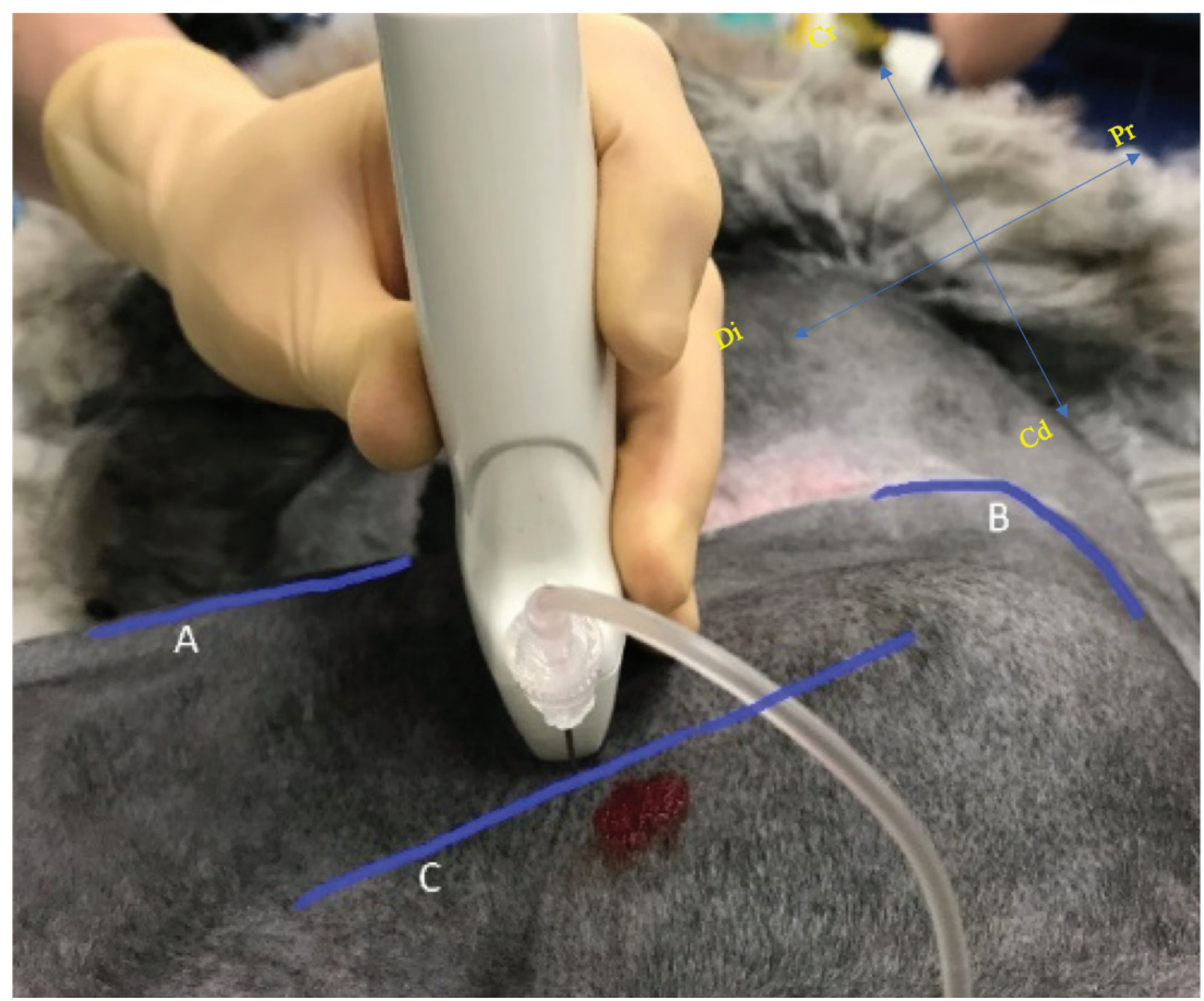

Fig. 1. Position of ultrasound transducer and needle performing the sciatic nerve block, identifying the cranial border of the limb (A), the wing of the ileum (B) and the orientation of the femur (C).

$\mathrm{mg} / \mathrm{kg}$ buprenorphine IV every 8 hours and $0.5 \mathrm{mg} / \mathrm{kg}$ meloxicam (Metacam ${ }^{\circledR} 20 \mathrm{mg} / \mathrm{ml}$, Boehringer Ingelheim, Berkshire, UK) IV once daily. Furthermore, the alpaca passed feces 30 minutes after the end of anesthesia and showed interest in food. Based on physical and behavioral assessment from a specialist farm animal clinician, pain was deemed to be well controlled. Buprenorphine was discontinued 24 hours following the end of anesthesia and meloxicam continued at the same dose. The patient remained in the hospital for 7 days for repeated wound assessment and redressing prior to discharge.

Case 2

A 5-year old male entire Suri Alpaca (V.pacos) weighing $80 \mathrm{~kg}$ was referred to the Royal Veterinary College for a left hind lateral claw removal, following a previous diagnosis of squamous cell carcinoma of the claw.

Pre-anesthetic examination was considered unremarkable. The patient was starved, a jugular catheter placed and premedication performed as for case 1 . The induction was performed 10 minutes later, with $3 \mathrm{mg} / \mathrm{kg}$ ketamine (Ketamidor ${ }^{\circledR} 100 \mathrm{mg}$ / $\mathrm{ml}$, Chanelle Pharma Group, Loughrea, Ireland) IV followed by intubation with a $12 \mathrm{~mm}$ ID cuffed endotracheal tube. The patient was connected to a circle rebreathing system and anesthesia was maintained with isoflurane in oxygen and air ( 0.8 and 0.2 , respectively). Hartmann's solution was infused at $2 \mathrm{ml} / \mathrm{kg} /$ hour. The animal was monitored as described in case 1 except for BP, which was measured invasively via a $20 \mathrm{~g}$ IV catheter placed in the right auricular artery. Mechanical ventilation was initiated immediately following the start of volatile anesthesia, with tidal volume and respiratory rate adjusted to maintain normocapnia.

The SN block was performed as described in case 1 , using $0.1 \mathrm{ml} / \mathrm{kg}(0.75 \mathrm{mg} / \mathrm{kg})$ of ropivacaine (Naropin ${ }^{\circledR}$ 0.75\%, AstraZeneca, Cambridge, UK). The patient was transferred to the surgical theatre where claw amputation was performed. General anesthesia lasted 1 hour and 40 minutes. During this time, the patient experienced mild hypotension with a mean arterial pressure of $60 \mathrm{mmHg}$. An IV bolus of $0.1 \mathrm{mg} /$ $\mathrm{kg}$ ephedrine (Ephedrine Hydrochloride, Martindale 


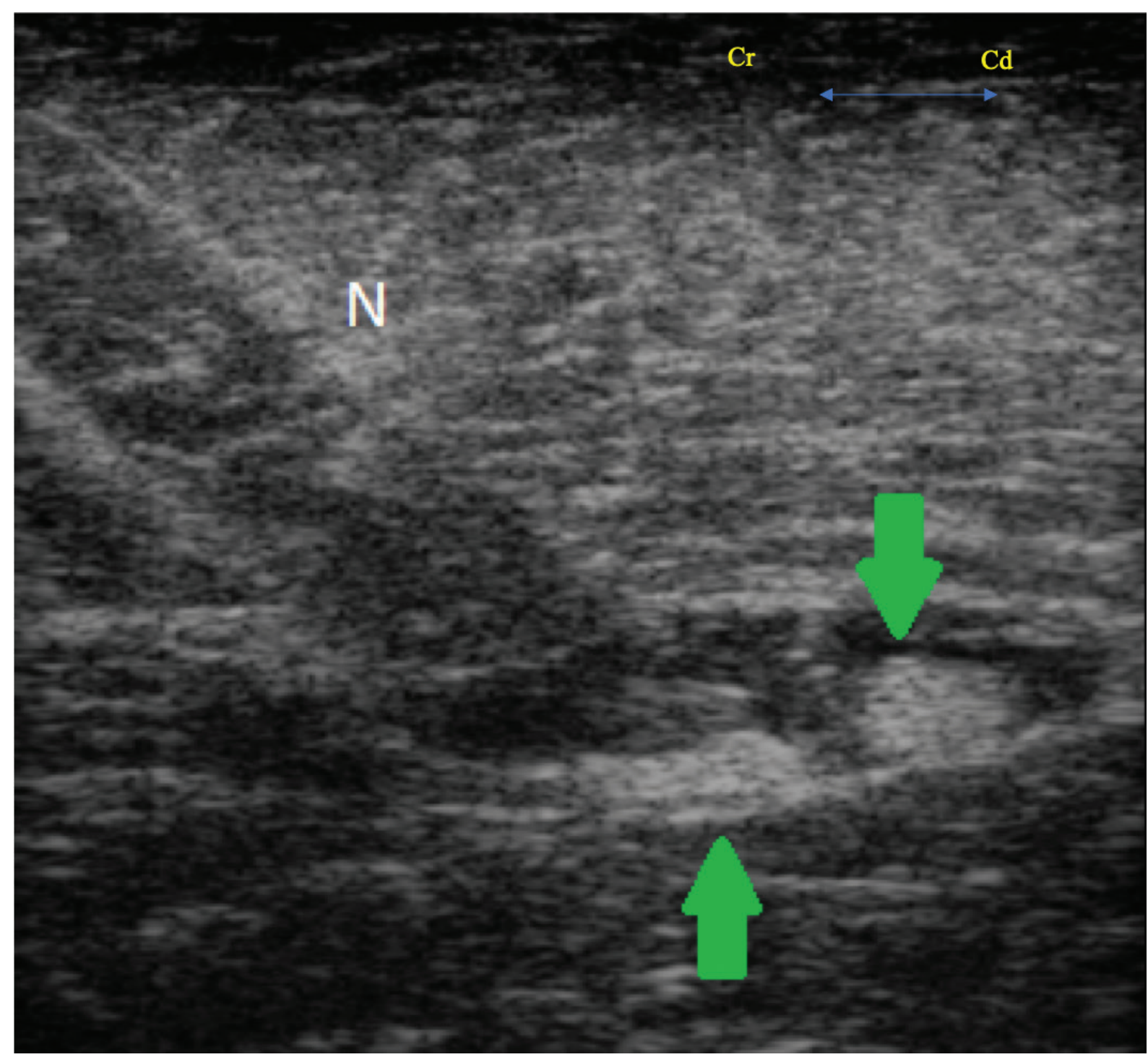

Fig. 2. Transverse ultrasound image showing the needle $(\mathrm{N})$ and the sciatic nerve (arrows), identified as two ellipsoid structures deep to the biceps femoris muscle.

Pharmaceuticals, Buckinghamshire, UK) was given, increasing mean BP to $\sim 95 \mathrm{mmHg}$. Further vasopressor administration was not required. No changes in HR, BP, or RR (suggestive of either sympathetic stimulation or nociception) were observed. The ETiso concentration ranged from $0.87 \%$ to $1.3 \%$ throughout.

At the end of the procedure, the patient experienced an uneventful recovery. Post-operative analgesia was provided with $0.01 \mathrm{mg} / \mathrm{kg}$ buprenorphine (Buprecare ${ }^{\circledR} 0.3 \mathrm{mg} / \mathrm{ml}$, Animalcare, York, UK) IV every 8 hours and $0.5 \mathrm{mg} / \mathrm{kg}$ meloxicam (Metacam ${ }^{\circledR} 20 \mathrm{mg} / \mathrm{ml}$, Boehringer Ingelheim, Berkshire, UK) IV once daily. The patient was standing, passing feces, and showing an interest in food 90 minutes following the end of anesthesia. Based on physical and behavioral assessment from a specialist farm animal clinician, pain was deemed to be well controlled. Buprenorphine was discontinued 24 hours later, while meloxicam continued at the same dose. Due to reduced interest in food 72 hours post-operatively, a blood sample was taken that showed an elevation in potassium $(5.9 \mathrm{mmol} / 1$; reference range $2.5-5.2)$, aspartate aminotransferase (1,114 U/l; reference range 175$340)$, creatine kinase (4,271 U/l; reference range 120-470), urea ( $23.5 \mathrm{mmol} / \mathrm{l}$; reference range $2.5-8.9)$, and creatinine (411 umol/l; reference range 53-194). At this stage, the patient was anuric and renal insufficiency was suspected; however, attempted medical treatment with IV isotonic fluid and IV furosemide administration was unsuccessful. Euthanasia was performed 6 days following surgery.

Post-mortem examination revealed reddened ileal and caecal mucosa, with fluid large intestinal contents. A moderate volume of serosanguineous peritoneal fluid was also present. On histopathology of the kidney, 


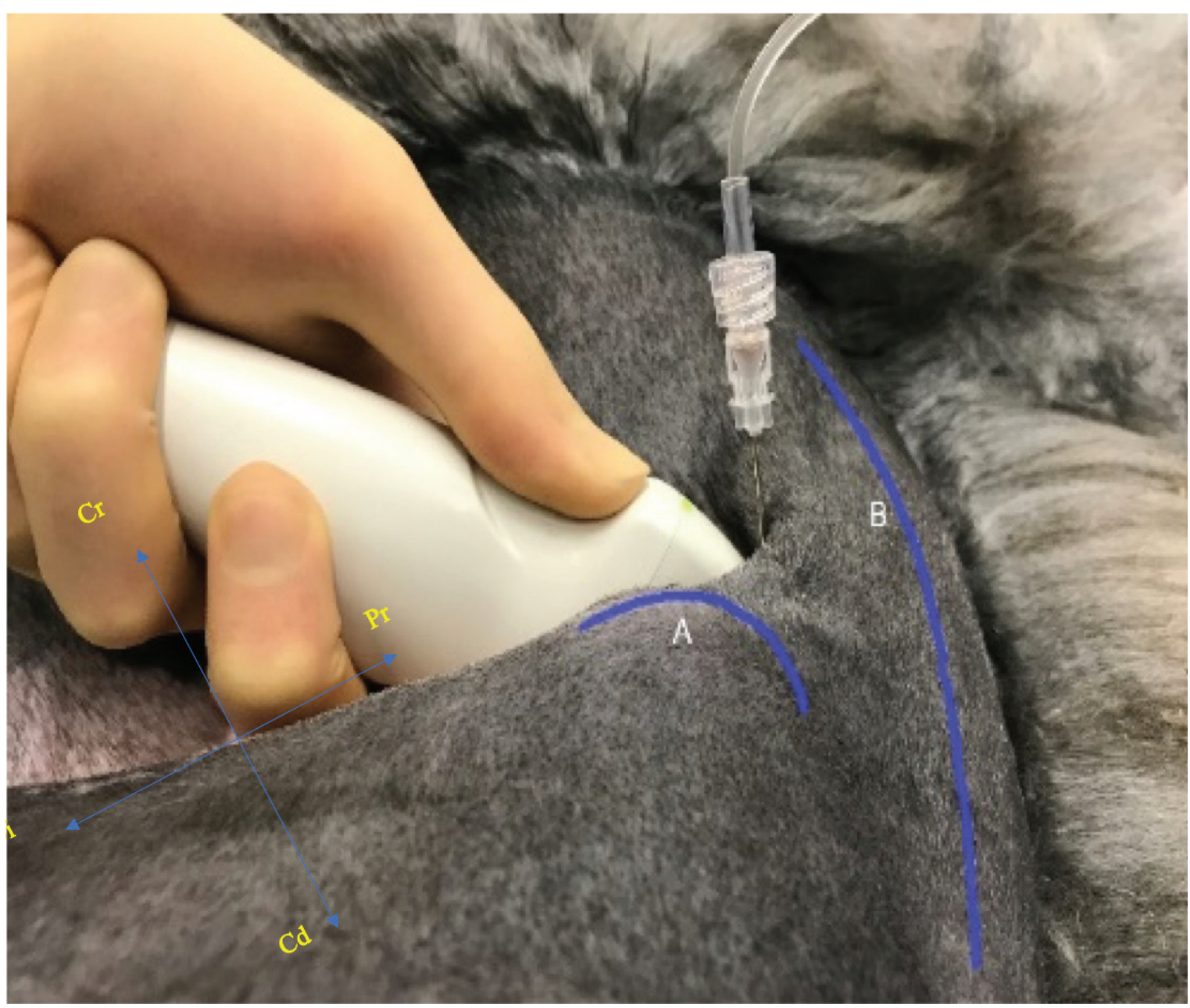

Fig. 3. Position of ultrasound transducer and needle performing the psoas compartment block, identifying the wing of the ilium (A) and the outline of the spine (B)

muscle, and liver, no signs of rhabdomyolysis were observed; however, changes were consistent with disseminated intravascular coagulation. This was likely secondary to bacterial toxins originating from the intestines. Renal insufficiency was most likely secondary to these findings.

\section{Ethical approval}

Data for these alpacas were collected from clinical cases and used retrospectively for publication. The alpacas included in this case report received a high standard of care and all the procedures carried out (including monitoring and blood sampling) were part of the routine care of animals undergoing anesthesia at our institution.

\section{Discussion}

Pain is poorly described in camelid species. It has been suggested that pain may result in side effects, such as decreased gut motility (Abrahamsen, 2009), as well as being considered a welfare issue. Conversely, the use of systemic analgesia, like opioids (Boscan et al.,
2006) or non-steroidal anti-inflammatory drugs (Radi, 2009), for example, may result in a number of adverse effects. Finding the correct approach to this clinical challenge may, therefore, be difficult. In humans, it is well established that a multimodal approach to analgesia with the use of different techniques and drugs with different mechanisms of action results in a number of benefits, from improved analgesia to a reduction of both the dose and possible side effects of the analgesics employed (Joshi et al., 2014). It is the authors' opinion that achieving multimodal analgesia utilizing LAs may have an important impact for species in which the knowledge of systemic analgesic drugs is still limited. For this reason, the authors attempted to extrapolate the techniques of the PsC and SN blocks (Echeverry et al., 2010; Tayari et al. 2017) routinely performed by the authors as a part of the anesthetic management of small animal patients at our institution.

In both cases in this report, the SN was easily identified with the aid of ultrasound and the spread of local anesthetic was clearly observed around the nerve 


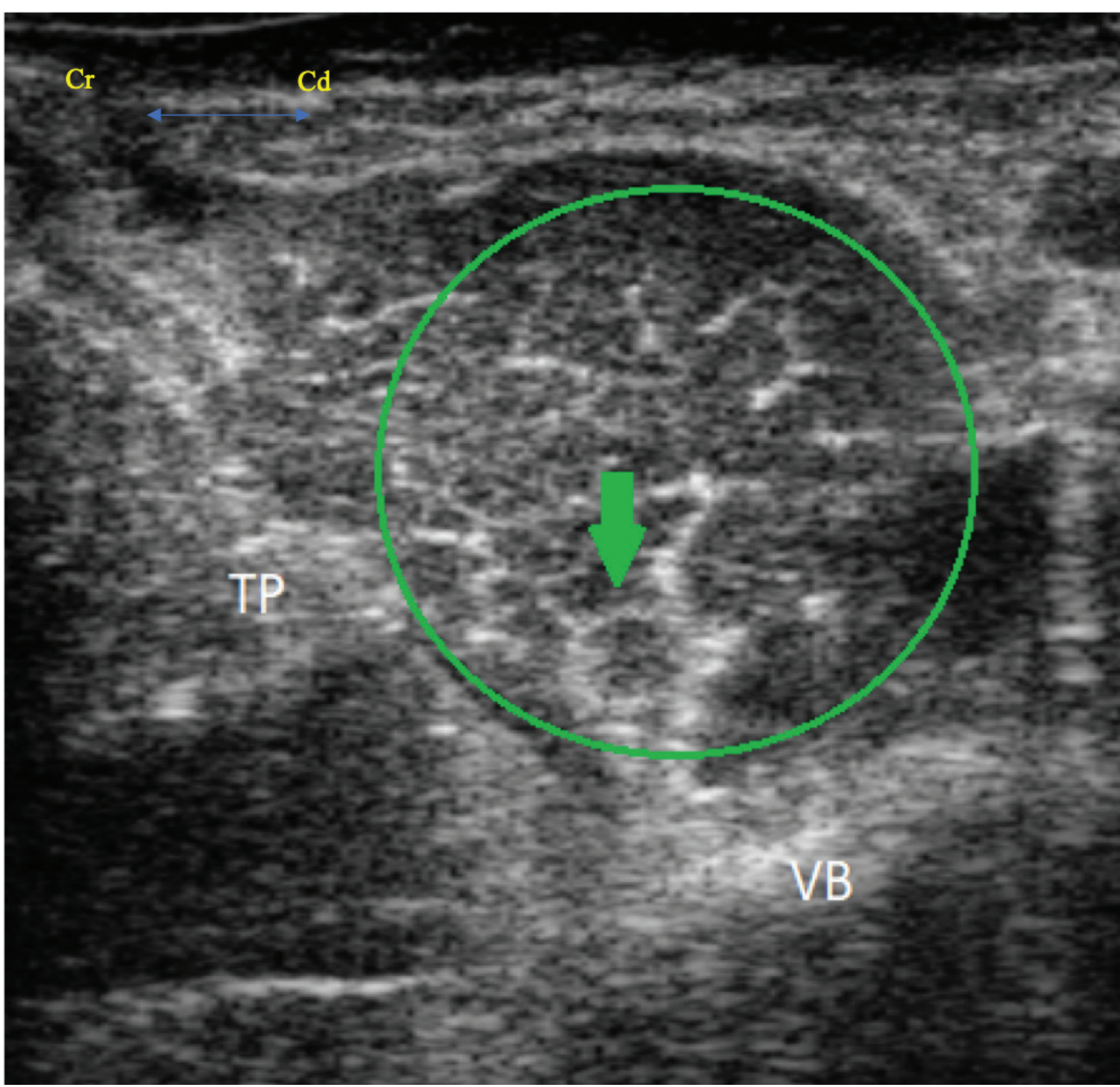

Fig. 4. Transverse ultrasound image showing the iliopsoas muscle (green circle), the femoral nerve (green arrow), the vertebral body (VB) and the transverse process (TP). The femoral nerve appears as a rounded hypoechoic structure with a thin hyperechoic rim.

following injection. However, interpretation of the psoas compartment was more complicated. While the identification of the IPM and FN was considered comparable to the canine models (Tayari et al., 2017), the ON was not clearly identifiable. Two main hypotheses can be made for this; 1 ) the relative small size of the $\mathrm{ON}$ in comparison to the FN or 2) the anatomical difference in the origin or trajectory of the $\mathrm{ON}$ through the psoas compartment. It is also worth mentioning that there is a lack of anatomical studies describing the specific innervation of the hind limb in the alpaca. While thought to be similar, it is possible that differences exist both between species and within the same species (a possibility for the cases in this report also). For instance, the ON was shown to provide innervation to the stifle in only $50 \%$ of the dog population (O'Connor and Woodbury, 1982). When differences across the species exist, the use of ultrasound-guided procedures permits the direct visualization of anatomical structures, therefore, partially overcoming this limitation. Furthermore, ultrasound guidance of a needle to these nervous structures, allows accurate application of LAs (Sites and Antonakakis, 2009) offering evaluable advantages in term of success rate and decreased incidence of complications, in comparison to either electro-location or blind techniques (Abrahams et al., 2009).

Ropivacaine $0.75 \%$ was the LA of choice for these cases. The authors made this decision based on clinical 
experience and the favorable pharmacological profile of the drug. Indeed, ropivacaine has a lower tendency to produce both motor block (Simpson et al., 2005) and cardiovascular or neurologic toxicity compared to other LAs, while it can still be considered a longer acting LA, like bupivacaine (Feldman et al., 1989).

It is worth mentioning that different techniques could have been used to provide similar effects for these cases. The first alternative to PNBs is the use of epidural anesthesia, something that has been reported for procedures of the proximal hind limb of the alpaca (Tee et al., 2005; Garcia Pereira et al., 2006; Abrahamsen, 2009). The decision was made not to use this technique for these cases, as in both situations recovery from anesthesia with rapid ambulation was deemed to be in the animals' best interests. An alternative PNB that could have been utilized is the FN block, at the level of the femoral triangle that has previously been investigated in farm animals (Adami et al., 2011; Viscasillas et al., 2015). Further options such as a ring block or a Bier block (Valverde and Sinclair, 2015), commonly used for procedures of the distal limb in farm animals, were considered for the second case. However, the authors concluded that lack of data illustrating the toxic dose of LAs in alpacas was a significant limitation to performing a Bier block. Second, placing an IV catheter in the distal limb of an alpaca can be extremely challenging (Plummer et al., 2013).

This report presents a few limitations worthy of discussion. First, the authors were unable to visualize the $\mathrm{ON}$ in the alpaca undergoing mid-femur amputation. Therefore, the advantages associated with block of the $\mathrm{ON}$ are considered speculation only with regards to this case.

Changes in HR, BP, and/or RR were utilized for the identification of nociception under general anesthesia. This is in accordance to a previous investigation (Adami et al., 2011) in which a deviation of 10\%, $25 \%$, and $50 \%$ in HR, mean arterial pressure (MAP), and $R R$ respectively, from the recorded baseline were considered suggestive of nociception. Based on our observations, one may assume that adequate analgesia had been achieved. However, physiological parameters can be influenced by a number of other factors including stress, disease status, and behavior of the animal Gleerup and Lindegaard, 2016.

Furthermore, the assessment of analgesia and nociception under general anesthesia were evaluated in the presence of concurrently administered systemic analgesics. Buprenorphine was given IV prior to surgery in both of these animals. Based on the experience of the authors and on published pharmacokinetics, buprenorphine is unlikely to result in adequate analgesia alone for either of the procedures performed. Indeed, due to the rapid clearance and short half-life (Dooley et al., 2017), buprenorphine may require frequent redosing to provide analgesia. It is, therefore, unlikely that buprenorphine resulted in prolonged analgesia for the entire intraoperative period for this case. This is also true for the ketamine administered; use of ketamine as an induction agent will also have provided analgesia in both cases, however, due to the length of the surgical procedures it was deemed at least unlikely to have played an ongoing analgesic role (Anderson, 2013). Importantly, difficulty exists in pain assessment of these animals as there are no validated pain scales for this species. Pain detection and quantification is mainly dependent on the ability of the investigator to detect behavioral changes, as for many prey animal species. In the immediate post-operative period, pain was evaluated hourly by either anesthetist or an expert farm animal clinician and was considered well controlled. With all of this information considered, both SN and PsC blocks may be considered effective adjuncts to systemic analgesia for this species.

In conclusion, this case report highlights the potential value of both ultrasound guided SN and PsC blocks for alpacas undergoing painful hind limb procedures, to achieve multimodal analgesia. Limitations to performing and accurately assessing their effectiveness still exist. With better understanding of the anatomy and through the creation of a validated pain scoring system, the possibility of species-specific loco-regional technique development exists.

\section{Conflicts of interest}

The authors declare that there is no conflict of interest. Author's contribution

Andrew Foster and Paolo Monticelli were the anaesthetists in charge of the cases. Alex McSloy was the primary clinician of the cases and carried out their clinical assessments. All the authors contributed, largely, to the writing and revision of this manuscript.

\section{References}

Abrahams, M.S., Aziz, M.F., Fu, R.F. and Horn, J.L. 2009. Ultrasound guidance compared with electrical neurostimulation for peripheral nerve block: a systematic review and meta-analysis of randomized controlled trials. Br. J. Anaesth. 102, 408-417.

Abrahamsen, E.J. 2009. Chemical restraint, anesthesia and analgesia for Camelids. Vet. Clin. North Am. Food Anim. Pract. 25, 455-494.

Adami, C., Bergadano, A., Bruckmaier, R.M., Stoffel, M.H., Doherr, M.G., and Spadavecchia, C. 2011. Sciatic-femoral nerve block with bupivacaine in goats undergoing elective stifle arthrotomy. Vet. J. $188,53-57$.

Aguiar, J., Mogridge, G. and Hall, J. 2014. Femoral fracture repair and sciatic and femoral nerve blocks in a guinea pig. J. Small Anim. Pract. 55(12), 635-639.

Anderson, D.E. 2013. Behaviour and capture techniques. In Veterinary techniques for Llamas and Alpaca, 1st ed. Eds., Anderson, D.E., Jones, M.L. and Meisner, M.D. Ames, IA: John Wiley \& Sons, Inc., pp: 1-7.

Boscan, P., Van Hoogmoed, L.M., Farver, T.B. and Snyder, J.R. 2006. Evaluation of the effects of the 
opioid agonist morphine on gastrointestinal tract function in horses. Am. J. Vet. Res. 67, 992-997.

Campoy, L. and Mahler, S. 2013. The pelvic limb. In Small animal regional anesthesia and analgesia, 1st ed. Eds., Campoy, L. and Read, M.R. Ames, IA: John Wiley \& Sons, Inc., pp: 199-226.

Campoy, L., Martin-Flores, M., Ludders, J.W, Erbb, H.N. and Gleeda, R.D. 2012. Comparison of bupivacaine femoral and sciatic nerve block versus bupivacaine and morphine epidural for stifle surgery in dogs. Vet. Anaesth. Analg. 39, 91-98.

Dooley, S.B., Aarnes, T.K., Lakritz, J., Lerche, P., Bednarski, R.M. and Hubbell, J.A.E. 2017. Pharmacokinetics and pharmacodynamics of buprenorphine and sustained-release buprenorphine after administration to adult alpacas. Am. J. Vet. Res. 78, 321-329.

d'Ovidio, D., Rota, S., Noviello, E., Briganti, A. and Adami, C. 2014. Nerve stimulator-guided sciaticfemoral block in pet rabbits (Oryctolagus cuniculus) undergoing hind limb surgery: a case series. J. Exot. Pet Med. 23, 91-95.

d'Ovidio, D., Noviello, E. and Adami, C. 2015 Nerve stimulator guided sciatic-femoral nerve block in raptors undergoing surgical treatment of pododermatitis Vet. Anaesth. Analg. 42, 449-453.

Echeverry, D.F., Francisco, G., Laredo, F., Ayala, M.D., Belda, E., Soler, M. and Agut, A. 2010. Ultrasoundguided block of the sciatic and femoral nerves in dogs: a descriptive study. Vet. J. 186, 210-215.

Feldman, H.S., Arthur, R. and Covino, B.G. 1989. Comparative systemic toxicity of convulsant and supraconvulsant doses of intravenous Ropivacaine, bupivacaine, and lidocaine in the conscious dog. Anesth. Analg. 69, 794-801.

Garcia Pereira, F.L., Greene, S.A., McEwen, M.M. and Keegan, R. 2006. Analgesia and anesthesia in camelids. Small Ruminant Res. 61, 227-233.

Gleerup, K.B. and Lindegaard, C. 2016. Recognition and quantification of pain in horses: A tutorial review. Equine Vet. Edu. 28, 47-57.

Haro, P., Laredo, F., Gil, F., Belda, E., Yala, M.D., Soler, M. and Agut, A. 2012. Ultrasound-guided dorsal approach for femoral nerve blockade in cats: an imaging study. J. Feline Med. Surg. 15, 91-98.

HuiChu, L. 2014. Perioperative monitoring and management of complications. In Farm animal anaesthesia: cattle, small ruminants, camelids, and pigs, 1st ed. Eds., HuiChu, L. and Walz, P. Ames, IA: John Wiley \& Sons, Inc., pp: 131.

Joshi, G.P., Schug, S.A. and Kehlet, H. 2014. Procedurespecific pain management and outcome strategies. Best Pract. Res. Clin. Anaes. 28, 191-201.

McNamee, D.A., Parks, L. and Milligan, K.R. 2002. Post-operative analgesia following total knee replacement: an evaluation of the addition of an obturator nerve block combined femoral and sciatic nerve block. Acta Anaesthesiol. Scand. 46, 95-99.
Monticelli, P., Campoy, L. and Adami, C. 2016. Ultrasound-guided femoral and sciatic nerve blocks for repair of tibia and fibula fractures in a Bennett's wallaby (Macropus rufogriseus). Case Rep. Anesthesiol. 16: 1-4.

O'Connor, B.L. and Woodbury, P. 1982. The primary articular nerves to the dog knee. J. Anat. 134, 563572.

Portela, D.A., Otero, P.E., Briganti, A., Romano, M., Corletto, F. and Breghi, G. 2013. Femoral nerve block: a novel psoas compartment lateral pre-iliac approach in dogs. Vet. Anaesth. Analg. 40, 194-204.

Plummer, P.J. and Schleining, J.A. 2013. Assessment and management of pain in small ruminants and camelids. Vet. Clin. North Am. Food Anim. Pract. 29, 185-208.

Radi, Z.A. 2009. Pathophysiology of Cyclooxygenase Inhibition in Animal Models. Toxicol. Pathol. 37, 34-46.

Shilo, Y., Pascoe, P.J., Cissell, D., Johnson, E.G., Kass, P.H. and Wisner, E.R. 2010. Ultrasoundguided nerve blocks of the pelvic limb in dogs. Vet. Anaesth. Analg. 37, 460-470.

Simpson, D., Curran, M.P., Oldfield, V. and Keating, G.M. 2005. Ropivacaine: a review of its use in regional anaesthesia and acute pain management. Drugs 65, 2675-2717.

Sites, B.D. and Antonakakis, J.G. 2009. Ultrasound guidance in regional anesthesia: state of the art review through challenging clinical scenarios. Local Reg. Anesth. 1, 1-14.

Tayari, H., Tazioli, G., Breghi, G. and Briganti, A. 2017. Ultrasound-guided femoral and obturator nerves block in the psoas compartment in dogs: anatomical and randomized clinical study. Vet. Anaesth. Analg. 44, 1216-1226.

Tee, S.Y., Dowling, B.A. and Dart, A.J. 2005 Treatment of long bone fractures in South American camelids: 5 cases. Aust. Vet. J. 83, 418-420.

Touray, S.T., de Leeuw, M.A., Zuurmond, W.W.A. and Perez, R.S.G.M. 2008. Psoas compartment block for lower extremity surgery: a meta-analysis. Br. J. Anaesth. 101, 750-760.

Valverde, A. and Sinclair, M. 2015. Ruminant and swine local anesthetic and analgesic techniques. In Lumb and jones veterinary anesthesia and analgesia, 5th ed. Eds., Grimm, K.A., Lamont L.A., Tranquilli W.J., Greene S.A. and Robertson S.A. Ames, IA: John Wiley \& Sons, Inc., pp: 941-959.

Vettorato, E., De Gennaro, C., Okushima, S. and Corletto, F. 2013. Retrospective comparison of two peripheral lumbosacral plexus blocks in dogs undergoing pelvic limb orthopaedic surgery. J. Small Anim. Pract. 54, 630-637.

Viscasillas, J., Drozdzynska, M.J. and McSloy, A. 2015. Ultrasound-guided sciatic and femoral nerve blocks for metatarsal fracture stabilisation in a twoday-old neonatal calf. Vet. Rec. 3, 1-4. 